EPJ Web of Conferences 61, 08004 (2013)

DOI: 10.1051/epjconf/ 20136108004

(C) Owned by the authors, published by EDP Sciences, 2013

\title{
The core shift measurements for two-sided jets affected by Free-Free absorp- tion using VLBA
}

\author{
Takafumi Haga ${ }^{1, a}$, Akihiro Doi ${ }^{1,2}$, Yasuhiro Murata ${ }^{1,2}$, Hiroshi Sudou ${ }^{3}$, Seiji Kameno ${ }^{4}$, Kazuhiro Hada ${ }^{5}$, and Hiroshi \\ Nagai $^{6}$ \\ ${ }^{1}$ Department of Space and Astronautical Science, The Graduate University for Advanced Studies (SOKENDAI) 3-1-1 Yoshinodai, \\ Chuo-ku, Sagamihara, Kanagawa 252-5210, Japan \\ ${ }^{2}$ Institute of Space and Astronautical Science, Japan Aerospace Exploration Agency 3-1-1 Yoshinodai, Chuo-ku, Sagamihara, \\ Kanagawa 252-5210, Japan \\ ${ }^{3}$ Department of Mathematical and Design Engineering, Faculty of Engineering, Gifu University 1-1 Yanagido, Gifu City 501-1193, \\ Japan \\ ${ }^{4}$ Joint ALMA Observatory, Alonso de Crdova 3107 Vitacura, Santiago, Chile \\ ${ }^{5}$ INAF Istituto di Radioastronomia via Gobetti 101, 40129 Bologna Italy \\ ${ }^{6}$ National Astronomical Observatory of Japan, 2-21-1 Osawa, Mitaka, Tokyo 181-858, Japan
}

\begin{abstract}
A radio core represents the peak of intensity in VLBI images and is located at the base of jets. It appears at different positions depending on frequencies. This is known as "core shift", caused by absorption of the core emission. The position of the central engine in an AGN can be estimated accurately by measuring the core shift with multifrequency and phase-referencing observations. We observed NGC 4261 using the VLBA at seven frequencies. This source is a nearby FR-I type radio galaxy at the distance of $30 \mathrm{Mpc}$ and has prominent two-sided jets. We measured the core shifts in not only approaching side but also counter side of the jets. The positions of core at infinity of frequency in both side indicated to come close asymptotically to the same position, which was separated by $82 \pm 16 \mu$ as from $43 \mathrm{GHz}$ core position, corresponding to $310 \pm 60$ $R_{\mathrm{S}}$ (Schwarzschild radius). This source also has another feature that there is a region affected by free-free absorption (FFA) in the vicinity of the core and toward the counter jet. Moreover, we also found the same feature in other three sources, $3 \mathrm{C} 84$, Cen A and Cyg A, which are also nearby galaxies with two-sided jets and with an indication of the FFA regions. We will measure the core shifts in these sources by using same technique as NGC 4261 in order to study the structure of circumnuclear plasma, to determine the position of the central engine and to test core shifts due to FFA.
\end{abstract}

\section{Introduction}

Very long baseline interferometry (VLBI), with the highest spatial resolution, is the sole technique to image the structure of central engines in AGNs. VLBI images of AGNs often shows a "radio core" that represents a compact unresolved component with the peak intensity at the base of jets. The position of the core appears different depending on frequencies due to absorption of the core emission, which is known as "core shift".

Hada et al. 2011[1] solved the frequency dependence of the core shift in M 87 using the multifrequency and phase-referencing observations. They determined that the limit of the core position as the frequency approaches infinity is located at about $14 R_{\mathrm{s}}$ away from the core at $43 \mathrm{GHz}$. Moreover, since this position indicated the position of the origin of the jet, they argued that the central engine is very close to the core at $43 \mathrm{GHz}$.

On the other hand, as noted by Marcsher et al. 2008[2], the position of the core at $43 \mathrm{GHz}$ in the BL Lac object

\footnotetext{
ae-mail: haga@vsop.isas.jaxa.jp
}

indicated to be standing shock point and then, the shock point in their model stay away by $10^{4}-10^{6} R_{\mathrm{s}}$ from the black hole on the basis of $\gamma$-ray monitoring observations [3]. Furthermore, the core shift was discovered at the downstream of jet in the BL Lac. BL Lac and other blazer sources also show that the position of the base of the jet is located on $10^{4} R_{\mathrm{s}}$ from $43 \mathrm{GHz}$ core position using the core shift measurements [4]. These examples indicates that It is difficult to determine the true position of the black hole using only the one-sided jet. Therefore, We will measure core shifts on two-sided jets in order to evaluate the position of the black hole.

\subsection{Our projects}

The targets are required to be nearby source because we have to measure core shifts accurately. We selected 4 sources, Centaurs A, NGC 4261, 3C 84, and Cygnus A. The distance of each source is listed in Table 1. Additionally, all of them have free-free absorption (FFA) feature around core. We can also investigate the effect of FFA on 
Table 1. The targets of our project

\begin{tabular}{lll}
\hline Object name & $\begin{array}{l}\text { Distance } \\
(\mathrm{Mpc})\end{array}$ & Evidence of FFA \\
\hline NGC 4261 & 31.6 & Jones et al. 2001[5] \\
3C 84 & 70 & Walker et al. 2000[6] \\
Cen A & 3.6 & Tingay et al. 2001[7] \\
Cyg A & 230 & Conway 1999[8] \\
\hline
\end{tabular}

the core shift. In this paper, we will show the results of NGC 4261, 3C 84, and Cen A.

\subsection{NGC 4261}

NGC 4261 (3C 270) is one of the nearby Fanaroff-Riley Class I (FR-I) radio galaxy. It has nearly symmetric kiloparsec-scale two-sided jets [9]. The distance of this galaxy is $31.6 \mathrm{Mpc}$ [10] corresponding to $0.15 \mathrm{pc} / \mathrm{mas}$. It is advantage for studying the regions close to the central engine within $1 \mathrm{pc}$. The nucleus of NGC 4261 is known to contain a central black hole with a mass of $(4.9 \pm 1.0) \times 10^{8} M_{\odot}[11]$ and a disk of gas and dust with a diameter of $\sim 300 \mathrm{pc}$, from the Hubble Space Telescope(HST) observations [12]. Previous VLBI studies showed that NGC 4261 also had a prominent two-sided jet to parsec scale along the large scale jets, which is parallel to the rotation axis of the dust disk [13]. One in west side was an approaching jet and another in east side was a receding, counter jet. The brightness both of approaching jet and counter jet was not so strongly affected by relativistic beaming. Piner et al. 2001[14] measured an apparent jet speed with the $8 \mathrm{GHz}$ VLBA monitor observations. From the jet-to-counterjet brightness ratio and the apparent speed, they estimated the intrinsic speed of jet was mild about $(0.46 \pm 0.02) c$ inclined by $63 \pm 3^{\circ}$ from the line of sight.

Another characteristic of NGC 4261 is the apparent discontinuity in the counter jet. This region called "gap", which was interpreted as the obscuration by edge-on, geometrically thin, cold and parsec scale disk with the temperature of $\sim 10^{4} \mathrm{~K}[5]$.

\section{Observations and Data reduction}

\subsection{Observations}

Multifrequency observations of NGC 4261 and $\mathrm{J} 1222+0413$ were conducted using the Very Long Baseline Array (VLBA) at seven bands, 1.4, 2.3, 5.0, and $8.4 \mathrm{GHz}$ on 2003 July 3 and 15, 22, and $43 \mathrm{GHz}$ on 2003 June 26 , separated by only 7 days each other. We used a phase-referencing technique to measure the radio core positions of NGC 4261 using first nodding relative to that of the nearby reference source, J1222+0413 separated within $2^{\circ}$ from NGC 4261; ...-T-C1-T-C1-T-..., where T and $\mathrm{C} 1$ represent the target and the primary calibrator, respectively. We repeated switching between the target and the calibrator by about 20 seconds scan. After we observed about 20 minutes at a frequency, we changed to next frequency. This resulted in achieving nearby the same $(u, v)$-covarage among frequencies which can minimize residual systematic errors in astrometry at different frequencies. The total on-source time on the target is about an hour at each frequency.

\subsection{Calibrations and Imaging}

We corrected antenna parallacitc angles, the Earth Orientation Parameters, and ionospheric dispersive delays using the ionospheric model from GPS data provided by the Jet Propulsion Laboratory (JPL) [15]. We used the data of 3C 273 for instrumental delays/phases and bandpass calibration. We performed a fringe-fitting on J1222+0413 as a phase calibrator $\mathrm{C}$, assuming a point source model. Subsequently, we corrected parameters for both the amplitude and the phase using a source structure model, which was established in the Difmap software [16] using deconvolution and self-calibration algorithms iteratively. We applied these solutions to the visivilities of NGC 4261 and obtained phase-referenced images of NGC 4261

\subsection{Core position measurement}

We measured the source position in phase-referenced images using JMFIT in AIPS, by a single ellipse gaussian profile model. However, we consider this source position was affected by blending a core and jets, and was slightly drifted toward the jet side relative to the true core position. We estimated this contribution by comparison between two ways of measuring core position in final images, which were performed on CLEAN and selfcalibration iteratively. The one is using JMFIT by one component and the other is using modelfit in Difmap to fit visibility data by multi circular gaussian components. Finally, we defined the core position as the position measured in phase-referenced images to be corrected for the jet contribution.

According to the method of Hada et al. 2011, we made error estimates of astrometric measurement, which consisted of ionospheric residuals, tropospheric residuals, antenna position error, Earth orientation error, apriori source coordinate error, core identification error and thermal noise error. These values are arranged inTable 4 . We assumed that total electron content (TEC) is $3 \times 10^{17} \mathrm{~m}^{-2}$ on the basis of GPS data and can be reduced to $1 / 4$ equivalent using TECOR in the ionospheric residuals [15]. A zenith excess path error is $2 \mathrm{~cm}$ in tropospheric residuals [17]. We adopted proper geometric errors like antenna error (3 mm), Earth orientation error (VLBA memo 69), and apriori source coordinate error from the source declination [18]. We estimated core identification error using the difference of core positions between the two different ways as mentioned above. The thermal noise error is defined by beam size over dynamic range of phase-referenced maps.

\section{Results}

Fig. 1 shows the (self-calibrated) CLEANed images with uniform weighting at every frequency. Approaching jets 


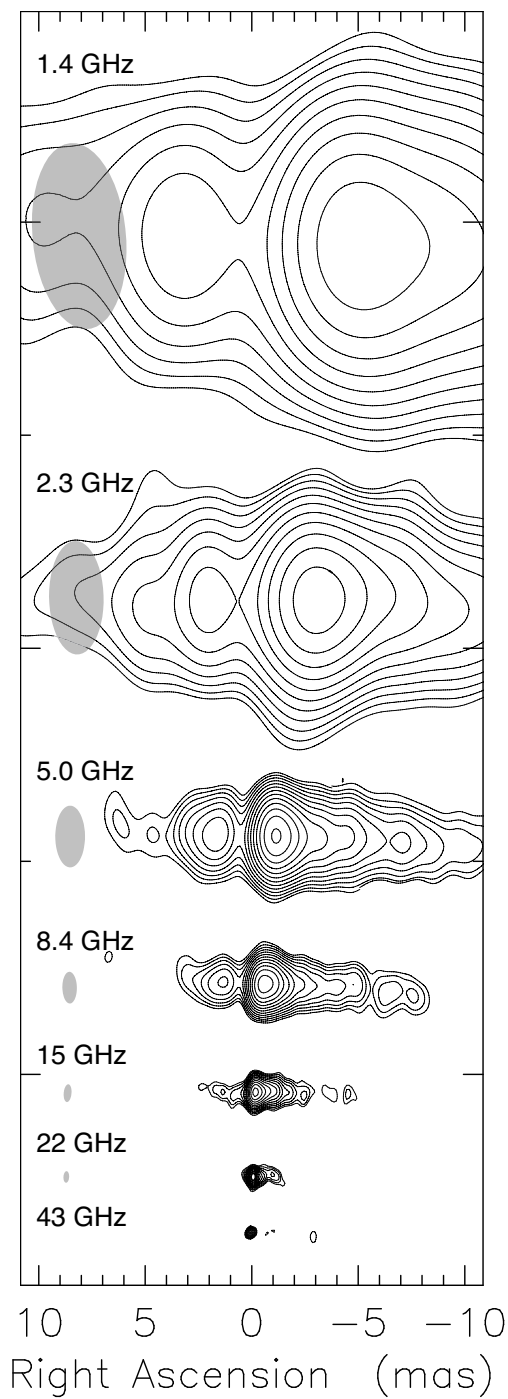

Figure 1. The self-calibrated images of NGC 4261 at every frequency, aligned relative to $43 \mathrm{GHz}$ core by taking account for core shifts. Synthesized beam size (FWHM), represented as gray ellipses.

and counter jets extending west and east from the brightness peak (core) appear in the images at $\leq 15 \mathrm{GHz}$. The image at $22 \mathrm{GHz}$ does not show the counter jet prominently, and the image at $43 \mathrm{GHz}$ does not show the jet like structure apparently. In fact, however, these images have model components using modelfit in Difmap in both approaching and counter jets. The lower the frequency, the more extended and the more symmetric the jets.

\subsection{Core shift measurement}

The core shift in the phase-referenced images of NGC 4261 appeared northwestward with respect to the core positions of $\mathrm{J} 1222+0413$. It is presumably the result of the combination of core shift originating in NGC 4261 and $\mathrm{J} 1222+0413$. The jet directions are almost perfectly perpendicular each other (east-west and north-south, respectively); therefore we can measure the core shifts of the both sources. The positions of the core on both sides
Table 2. The positions of the core. $r_{\mathrm{AJ}}$ and $r_{\mathrm{CJ}}$ represent the core position in approaching jet and counter jet, respectively.

\begin{tabular}{crr}
\hline $\begin{array}{c}v \\
(\mathrm{GHz})\end{array}$ & $\begin{array}{r}r_{\mathrm{AJ}} \\
(\mathrm{mas})\end{array}$ & $\begin{array}{r}r_{\mathrm{CJ}} \\
(\mathrm{mas})\end{array}$ \\
\hline 1.4 & $-4.92 \pm 2.06$ & $4.74 \pm 2.05$ \\
2.3 & $-2.98 \pm 0.79$ & $2.73 \pm 0.79$ \\
5.0 & $-1.18 \pm 0.17$ & $1.97 \pm 0.17$ \\
8.4 & $-0.52 \pm 0.08$ & $1.60 \pm 0.08$ \\
15 & $-0.21 \pm 0.03$ & $0.73 \pm 0.33$ \\
22 & $-0.12 \pm 0.02$ & $0.29 \pm 0.31$ \\
43 & $0 \pm 0.02$ & $0.17 \pm 0.03$ \\
\hline
\end{tabular}

Table 3. The parameters of the core shift fitting

\begin{tabular}{cccc}
\hline & $\Omega$ & $k$ & $c$ \\
\hline$F_{1}(v)$ & $-8.42 \pm 0.86$ & $-1.22 \pm 0.06$ & $0.082 \pm 0.016$ \\
$F_{2}(v)$ & $6.86 \pm 0.26$ & $-1.15 \pm 0.06$ & $0.081 \pm 0.017$ \\
\hline
\end{tabular}

at all frequency are arranged in Tab.2. Fig.2 shows that core shifts of NGC 4261 relative to $43 \mathrm{GHz}$ core are plotted at all the other frequency. We determined the best fit curve by least-square method with a power-law function of frequencies,

$$
F(v)=\Omega \cdot v^{k}+c,
$$

where $\Omega, c$ and $k$ are free parameters of fitting and represent a scale factor, a power low index and an asymptotic line at infinity of frequency, respectively. In approaching jet side, these values are determined to be $\Omega=$ $-8.42 \pm 0.86, k=-1.22 \pm 0.06$, and $c=0.082 \pm 0.016 \mathrm{re}-$ spectively. From our result, the asymptotic parameter was determined within a tolerance of $32 \mu$ as and separated by $82 \mu$ as from $43 \mathrm{GHz}$ core position, which corresponds to $310 \pm 60 R_{\mathrm{s}}$. Moreover, we also measured the core positions in counter jet side of NGC 4261. In this paper, we defined the core of the counter jet as a closest and east side component to the core of approaching jet, which have maximum intensity using modelfit in Difmap. We performed to fit the core of counter jet. However, three parameters had too large errors to fit when all parameter was free. Therefore, we tried to fit them again in two ways with other assumptions, which will be discussed in later in section 4.2 .

\subsection{Spectral index}

Spectral index $\left(\alpha, S \propto v^{\alpha}\right)$ maps were produced from final CLEANed images of two adjacent frequency using the AIPS task COMB at every frequency pair. In order to make accurate spectral index maps, the images between different frequencies were aligned on the basis of phase reference astrometry and we used the same beam equivalent to synthesized beam at the lower frequency .

Fig. 3 shows the spectral index maps between 1-2 GHz pair and 5-8 GHz. The region of $\alpha>+2.5$ in these maps besides the $8-15 \mathrm{GHz}$ pair, suggesting FFA due to a circumnuclear absorber. 


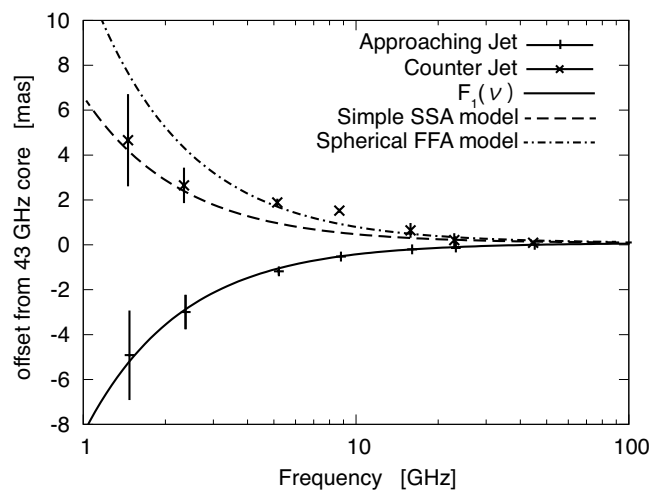

Figure 2. Bar and cross marks are the core positions of the approaching jet and the counter jet in NGC 4261. Some lines are functions fitted by using least-square method. In counter jet side, we estimated the parameters in two cases. One is SSA model and another is spherical FFA plasma model. The value of $k$ and $c$ are equivalent to them of $F_{1}(v)$, assuming the jet inclination, we calculated $\Omega$ of counter jet side in two case using one in the approaching jet side.

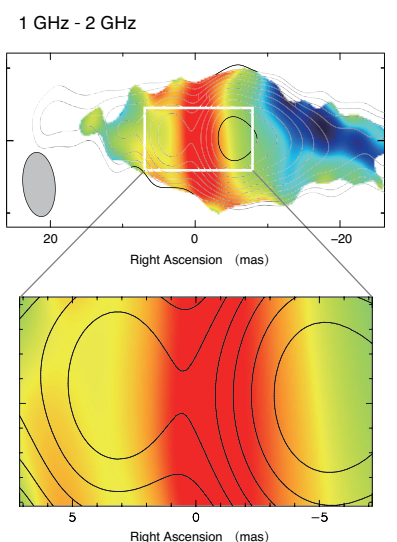

$5 \mathrm{GHz}-8 \mathrm{GHz}$

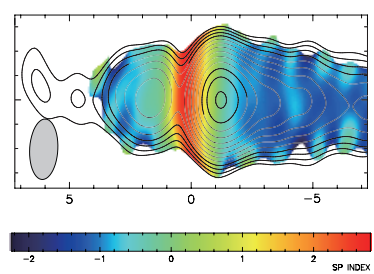

Figure 3. The spectral index maps between $1 \mathrm{GHz}$ and $2 \mathrm{GHz}, 5 \mathrm{GHz}$ and $8 \mathrm{GHz}$ synthesized after we restore the images with the lower frequency beam. At lower frequency pair, the spectral index in the part covering \pm 10 mas shows inverted spectral, indicating the absorption and the maximum of $\alpha$ reaches 3.5. At higher frequency pair, the part of $\alpha>2.5$ was located on 0.4 mas to east jet side and the position of the spectral index maximum was also located on the counter jet.

\section{Discussion}

\subsection{BH position in NGC 4261}

Fig. 4 shows that the core (the most upstream component) in counter jet side exists at $0.17 \pm 0.03$ mas $\left(\approx 640 \pm 110 R_{\mathrm{s}}\right)$ from the core at $43 \mathrm{GHz}$. The position of the black hole needs to be located within at least $750 R_{\mathrm{S}}$ because it should be located between the cores of both sides jet. Moreover the origin of the jet in counter jet side is coincident with that of the other side (and we will discuss later). It indicates that the assumption that the origin of a jet coincides with the position of the black hole is reasonable for NGC 4261 .

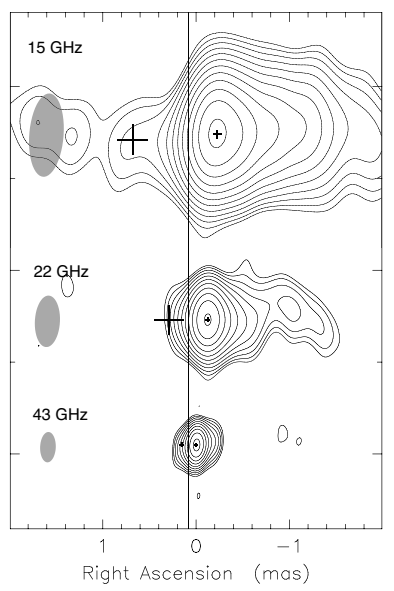

Figure 4. The extended image of Fig. 1 at $v \geq$ $15 \mathrm{GHz}$ within \pm 2 mas. The cross marks show the position of the core on each jet side. The line at 0.082 mas represents the position of the black hole. We can distinguish precisely between the position of the black hole and the cores at both side with high positional accuracy.

\subsection{The interpretation for core shift}

It is considered that the physical processes of causing the core shift are SSA and/or FFA. We will find what model can explain the core shift of NGC 4261 to focus on the results of the fitting parameters in the equation (1). Although we could not fit the core shift on counter jet side closely, we can estimate the parameters of the counter jet side using those of the other side at each model.

One is a pure SSA model. According to Lobanov 1998 [19], the core shift from the origin of a jet can be described as

$$
\Delta r_{\text {mas }} \approx \frac{C_{\mathrm{r}}(1+z)}{D_{L} \gamma^{2} \phi_{0} \nu_{1}}\left[\frac{L_{\mathrm{syn}} \sin \theta}{\beta(1-\beta \cos \theta) \Theta}\right]^{2 / 3},
$$

with $C_{\mathrm{r}}=4.56 \times 10^{-12}$, and where $\gamma \equiv 1 / \sqrt{1-\beta^{2}}$, $\beta \equiv v / c, \phi_{0}$, and $\theta$ are Lorentz factor, an intrinsic jet velocity, a jet opening angle, and an inclination, respectively. $D_{\mathrm{L}}$ and $L_{\text {syn }}$ are a luminosity distance to the source given in parsec, and a synchrotron luminosity in erg/s. Then, $\Theta$ is connected to the structure of the jet like conical or paraboloidal jet and the value is no more than $\ln 3$ for most of parsec-scale jet.

Then, we will predict the core shift profile in counter jet side for SSA case. Assuming intrinsically symmetric jets, $k$ and $c$ in counter jet side should have the same values. However, as compared between the equations in (1) and (2), you find $\Omega$ depends on geometrical parameters as well as intrinsic parameters of a jet. The only difference of $\Omega$ between approaching and counter jet depend on inclination, $\theta$. Therefore the value of $\Omega$ between both sides need to satisfy $\Omega_{\mathrm{AJ}}>\Omega_{\mathrm{CJ}}$. It indicates that the position of the core at same frequency is farther away from the black hole in the approaching jet than that in counter jet. The $\Omega_{\mathrm{CJ}}$ can be obtained using $\Omega_{\mathrm{AJ}}$ and the jet inclination of NGC 4261. The curve predicted by a pure SSA model in Fig. 2 can explain the position of cores at low frequency $\leq$ $2 \mathrm{GHz}$ and high frequency $\geq 22 \mathrm{GHz}$. On the other hand, the observed position of the core at 5,8 and $15 \mathrm{GHz}$ are significantly farther way than the predict line. It indicates that a SSA model solely lacks a sufficient absorption to explain the core shift.

Another is a FFA model. From the results of the spectral index maps, the regions near the black hole indicated the presence of the FFA plasma. We suppose the spherical 
FFA plasma for simplicity by the parameters of the core shift measurement as well. In this case, $k$ is dependent the structure of the spherical FFA plasma.

As the case in SSA, $k$ should become also common value in both side if FFA absorber has the symmetric structure like a spherical symmetric. Then, $\Omega$ of the FFA case reflects the path length of the absorber, that is, the structure of the absorber (and discuss about details in Appendix section). Suppose the absorber cover counter jet side with disk like or spherical like structures, the value of $\Omega$ will show $\Omega_{\mathrm{AJ}}<\Omega_{\mathrm{CJ}}$ because the path length of counter jet side is longer than that of the other side. The curve predicted by a spherical FFA plasma model in Fig.2, can interpret the result of core shifts at frequency more than $5 \mathrm{GHz}$ on counter jet side. However, the core shifts at 1 and $2 \mathrm{GHz}$ are inconsistent because the amount of them are smaller than the expectation.

Therefore, both of these simple models cannot describe the core shift in two side at once. A possible solution is the SSA core shift in addition to an absorbed component, which compensates for the inadequate core shift. In fact, our results shows that the positions of the core on counter jet side are larger than the other side at 5, 8, and $15 \mathrm{GHz}$ over the range of error. It suggests the existence of an absorber. Moreover, when we perform fitting cores shift without the cores at these frequencies, the best fitted values of $k_{2}$ and $c_{2}$ become almost equal to those of the approaching side (Table 3 ) as expected from the SSA theory. It indicates that the core at 5,8 , and $15 \mathrm{GHz}$ are strongly affected by FFA and it is consistent with the results of the spectral index maps showing FFA regions at these frequency as previous studies [5]. Fig.5 shows our model to explain core shift and spectral index.

\section{Summary}

We measured the origin of NGC 4261's jet as the core at infinity of frequency using core shift profile, which is separated by $82 \pm 16 \mu$ as from $43 \mathrm{GHz}$ core position, corresponding to $310 \pm 60 R_{\mathrm{s}}$. In addition, we also measured the core shift in counter jet side and confirmed that the positions of core at infinity of frequency in both side indicated to come close asymptotically to the same position. Therefore, we conclude that the jet origin estimating by core shift measurements coincide with the position of the black hole

The spectral index maps shows the possibility of FFA, $\alpha>2.5$ in counter jet side. The core shift profile in counter jet side cannot be explained for a synchrotron self absorption. and it needs an absorbed component with disk like structure.

We are investigating other souces. Fig.6 and Fig.7 show the (self-calibrated) CLEANed images of 3C 84 and Cen A. We will measure the core shift in order to determine the black hole position, and study the structure of FFA plasma by comparing with NGC 4261.

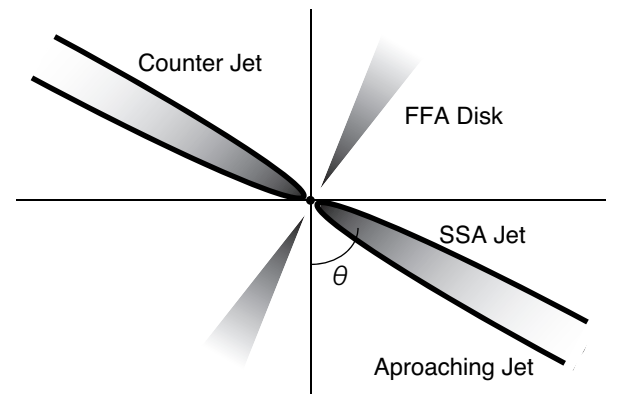

Figure 5. Schematic illustration of our model which enables to explain the core shift in both side jets at once.
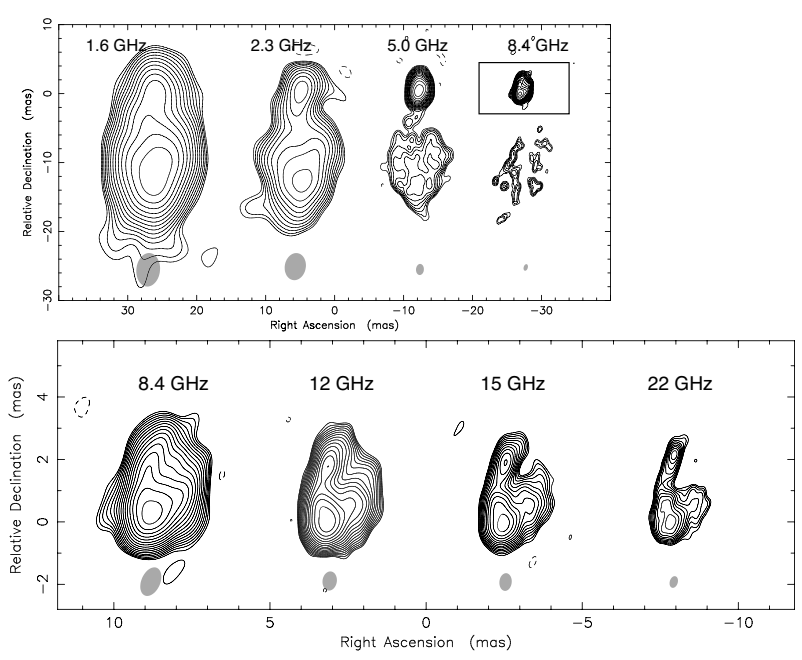

Figure 6. The CLEANed images of 3C 84. The gray ellipses represent the synthesized beam size (FWHM).

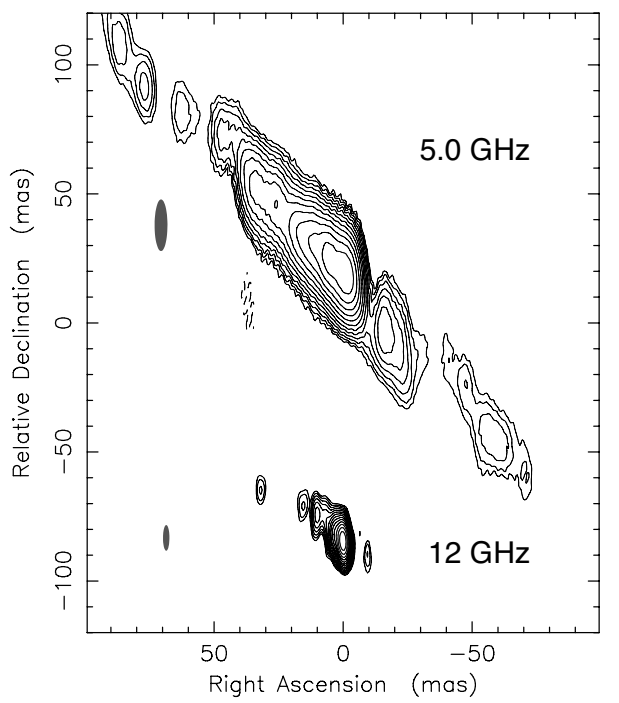

Figure 7. The CLEANed images of Cen A at 5 and $12 \mathrm{GHz}$. The gray ellipses on the left represent the synthesized beam size (FWHM). 
EPJ Web of Conferences

Table 4. The error budget

\begin{tabular}{lccccccc}
\hline Frequency [GHz] & 1.4 & 2.3 & 5.0 & 8.4 & 15 & 22 & 43 \\
\hline error components (common) $(\mu \mathrm{as})$ & & & & & & & \\
Beam width/ signal to noise & 52 & 24 & 10 & 6 & 4 & 3 & 2 \\
Ionosphere & 2006 & 779 & 161 & 57 & 17 & 8 & 2 \\
Troposphere & 13 & 13 & 13 & 13 & 13 & 13 & 13 \\
Earth orientation & 5 & 5 & 5 & 5 & 5 & 5 & 5 \\
Antenna position & 2 & 2 & 2 & 2 & 2 & 2 & 2 \\
Apriori source coordinates & 1 & 1 & 1 & 1 & 1 & 1 & 1 \\
\hline Core identification $(\mu$ as) & & & & & & & \\
A-Jet & 349 & 60 & 17 & 40 & 0.2 & 5 & 3 \\
C-Jet & 343 & 26 & 5 & 50 & 326 & 312 & 19 \\
\hline Total error (RSS) ( $\mu$ as) & & & & & & & \\
A-Jet & 2055 & 792 & 170 & 77 & 30 & 23 & 19 \\
C-Jet & 2054 & 790 & 169 & 82 & 328 & 313 & 25 \\
\hline
\end{tabular}

\section{References}

[1] Hada, K., Doi, A., Kino, M., et al., Nature, 477, 185 (2011)

[2] Marscher, A. P., Jorstad, S. G., D’Arcangelo, F. D., et al., Nature, 452, 966 (2008)

[3] Albert, J., Aliu, E., Anderhub, H., et al., ApJ, 666, L17 (2007)

[4] O’Sullivan, S. P., \& Gabuzda, D. C., MNRAS, 400, 26 (2009)

[5] Jones, D. L., Wehrle, A. E., Piner, B. G., \& Meier, D. L., ApJ, 553, 968 (2001)

[6] Walker, R. C., Dhawan, V., Romney, J. D., Kellermann, K. I., \& Vermeulen, R. C., ApJ, 530, 233 (2000)

[7] Tingay, S. J., \& Murphy, D. W., ApJ, 546, 210 (2001)

[8] Conway, J. E., Highly Redshifted Radio Lines, 156, 259 (1999)

[9] Birkinshaw, M., \& Davies, R. L., ApJ, 291, 32 (1985)
[10] Tonry, J. L., Blakeslee, J. P., Ajhar, E. A., \& Dressler, A., ApJ, 530, 625 (2000)

[11] Ferrarese, L., Ford, H. C., \& Jaffe, W., ApJ, 470, 444 (1996)

[12] Jaffe, W., Ford, H. C., Ferrarese, L., van den Bosch, F., \& O'Connell, R. W., Nature, 364, 213 (1993)

[13] Jones, D. L., Wehrle, A. E., Meier, D. L., \& Piner, B. G., ApJ, 534, 165 (2000)

[14] Piner, B. G., Jones, D. L., \& Wehrle, A. E., AJ, 122, 2954 (2001)

[15] Mannucci, A. J., Iijima, B., Sparks, L., et al., Journal of Atmospheric and Solar-Terrestrial Physics, 61, 1227 (1999)

[16] Shepherd, M. C., Pearson, T. J., \& Taylor, G. B., BAAS, 26, 987 (1994)

[17] Beasley, A. J., \& Conway, J. E., Very Long Baseline Interferometry and the VLBA, 82, 327 (1995)

[18] Pradel, N., Charlot, P., \& Lestrade, J.-F., A\&A, 452, 1099 (2006)

[19] Lobanov, A. P., A\&A, 330, 79 (1998) 